Proc. Indian Acad. Sci. (Math. Sci.), Vol. 104, No. 3, August 1994, pp. 581-591.

(C) Printed in India.

\title{
Extended Kac-Akhiezer formulae and the Fredholm determinant of finite section Hilbert-Schmidt kernels
}

\author{
S GANAPATHI RAMAN and R VITTAL RAO \\ Department of Mathematics, Indian Institute of Science, Bangalore 560012, India \\ MS received 29 October 1993; revised 15 February 1994

\begin{abstract}
This paper deals with some results (known as Kac-Akhiezer formulae) on generalized Fredholm determinants for Hilbert-Schmidt operators on $L_{2}$-spaces, available in the literature for convolution kernels on intervals. The Kac-Akhiezer formulae have been obtained for kernels which are not necessarily of convolution nature and for domains in $\mathbb{R}^{n}$.
\end{abstract}

Keywords. Hilbert-Schmidt integral operator; Fredholm determinant; Kac-Akhiezer formula.

\section{Introduction}

The classical Fredholm determinant [2] of a given symmetric Hilbert-Schmidt integral (briefly $\mathrm{H}-\mathrm{S}$ ) operator $T$ is an analytic function $D_{T}(\lambda)$ with the property that $\lambda(\neq 0)$ is a zero of $D_{T}(\lambda)$ if and only if $1 / \lambda$ is an eigenvalue of $T$. We shall call any analytic function $f_{T}(\lambda)$, as a Fredholm determinant of $T$ if its zeros are precisely reciprocals of the non-zero eigenvalues of $T$.

For convolution kernels such a Fredholm determinant was obtained by Kac [3], [4] and Akhiezer [1]. Their result is briefly summarized below:

Let $k(x)$ satisfy the following conditions:

1. $k(x)$ is a bounded, continuous, real valued function on $(-\infty, \infty)$,

2. $k(x)=k(-x)$,

3. $\hat{k} \in L_{1}\left(\mathbb{R}^{1}\right)$, where $\hat{k}$ is the Fourier transform of $k$,

4. $\int_{-\infty}^{\infty}|x k(x)| \mathrm{d} x<\infty$,

and

5. $\int_{-\infty}^{\infty}|x k(x)| \mathrm{d} x<1 / 2$.

Then the Fredholm determinant $D_{T}(\lambda)$ of the symmetric H-S operator

$$
T_{\tau} f=\int_{0}^{\imath} k(x-y) f(y) \mathrm{d} y
$$

on $L_{2}[0, \tau]$ is given by

$$
D_{\tau}(\lambda)=\prod_{j=1}^{\infty}\left[1-\lambda \lambda_{i}(\tau)\right]=\exp \left[-\int_{0}^{\tau} \alpha(0, s, \lambda) \mathrm{d} s\right],
$$


where $\alpha(x, \tau, \lambda)$ is the solution of the integral equation:

$$
\alpha(x, \tau, \lambda)=\lambda k(x)+\lambda \int_{0}^{\tau} k(x-y) \alpha(y, \tau, \lambda) \mathrm{d} y .
$$

We shall refer to (1.1) as the Kac-Akhiezer formula.

In the above result we observe that the main assumptions on the kernel are:

(i). $k(x)$ is a continuous function on $(-\infty, \infty)$;

(ii). The domain of the integral is an interval in $\mathbb{R}^{1}$;

(iii). The kernel is of convolution type.

This result has been extended for the case when $k(x)$ is only continuous in a neighbourhood of the origin. Further, when $k(x)$ is not continuous near the origin, a meromorphic function whose poles and zeros correspond to the eigenvalues has been obtained in [5], [7]. Subsequently Rao and Sukavanam [9] have extended these results for normal integral operators. But all these results are with the assumptions (ii) and (iii).

The aim of this paper is to obtain these results by replacing (ii) and (iii) by (ii)' and (iii)' below, where (ii)' is essentially replacing the interval by a suitable smooth domain in $\mathbb{R}^{n}$ and (iii)' is essentially replacing the convolution nature of the kernel by an assumption that the map $x \mapsto k(x, \cdot)$ is continuous.

(ii): $\mathscr{C}_{1}$ : For every $\tau \geqslant 0, \Omega_{\varepsilon}$ is a bounded subset of $\mathbb{R}^{n}$.

$\mathscr{C}_{2}:\{0\}=\Omega_{0} \subseteq \boldsymbol{\Omega}_{\tau_{1}} \subseteq \Omega_{\mathrm{s} 2}$ if $0 \leqslant \tau_{1} \leqslant \tau_{2}$, where 0 is the zero vector in $\mathbb{R}^{n}$.

$\mathscr{C}_{3}$ : The map $\tau \mapsto \rightarrow \mu\left(\bar{\Omega}_{\tau}\right)$ from the interval $[0, \infty)$ to $\mathbb{R}^{1}$ is absolutely continuous in every bounded interval in $[0, \infty)$, where $\mu$ is the Lebesgue measure on $\mathbb{R}^{n}$ and $\bar{\Omega}_{\tau}$ is the closure of $\Omega_{\tau}$ with respect to the standard topology of $\mathbb{R}^{n}$.

$\mathscr{C}_{4}$ : For every $\tau \geqslant 0$ there exists a surface measure $\sigma_{\tau}$ on $\partial \bar{\Omega}_{\imath}$, such that

(i). $g(t) \stackrel{\text { def }}{=} \int_{\partial \bar{\Omega}_{\tau}} f(x) \mathrm{d} \sigma_{\imath}(x)$ is an integrable function on $[0, \tau]$ for $f \in C^{0}\left(\bar{\Omega}_{\tau}\right)$, where $C^{0}\left(\bar{\Omega}_{\tau}\right)$ is the space of all continuous functions on $\bar{\Omega}_{\tau}$, and

(ii). $\int_{\overline{\mathbf{\Omega}}_{\mathrm{r}}} f(x) \mathrm{d} x=\int_{t=0}^{\tau}\left[\int_{\partial \overline{\mathbf{\Omega}}_{\mathrm{t}}} f(x) \mathrm{d} \sigma_{\mathrm{z}}(x)\right] \mathrm{d} t, \quad \forall f \in C^{0}\left(\overline{\mathbf{\Omega}}_{\tau}\right)$.

(A simple motivation for such a family $\left\{\Omega_{\tau}\right\}_{\tau \geqslant 0}$ in $\mathbb{R}^{n}$ is the ball in $\mathbb{R}^{n}$ of radius $\tau$ and centre at $\mathbf{0}$.)

(iii)': The kernel is such that

$\mathscr{C}_{5}: k(x, y)$ is defined in $\mathbb{R}^{n} \times \mathbb{R}^{n}$, and is symmetric.

$\mathscr{C}_{6}: k$ is locally square integrable.

$\mathscr{C}_{7}:$ For every $x \in \mathbb{R}^{n}, k(x, y)$ is well defined and is in $L_{2}\left(\mathbb{R}^{n}\right)$, and for any two compact subsets $E, F$ of $\mathbb{R}^{n}$, the mapping $x \mapsto k_{x}$ from $E$ into $L_{2}(F)$ is continuous; where $k_{x}(\cdot)=k(x, \cdot)$.

By $\mathscr{C}_{5}$ and $\mathscr{C}_{6}$ it follows that for every $\tau>0$.

$$
\left(K_{\mathfrak{\tau}} f\right) x=\int_{\overline{\mathbf{\Omega}}_{\mathrm{r}}} k(x, y) f(y) \mathrm{d} y \quad\left(f \in L_{\mathbf{2}}\left(\overline{\mathbf{\Omega}}_{\mathrm{\tau}}\right)\right)
$$

defines a compact self adjoint operator on $L_{2}\left(\bar{\Omega}_{\tau}\right)$. Further we assume 
$\mathscr{C}_{8}:$ For all $\tau>0,1$ is not in the spectrum of $K_{\tau}$.

Then $\left(I-K_{\tau}\right)^{-1}$ exists for every $\tau>0$ where $I$ is the identity operator on $L_{2}\left(\bar{\Omega}_{\tau}\right)$.

Let $\alpha_{1}(x, y, \tau)$ denote the unique solution of the equation

$$
\alpha(x, y, \tau)=k(y, x)+\int_{\bar{\Omega}_{\mathfrak{\tau}}} k(y, z) \alpha(x, z, \tau) \mathrm{d} z
$$

and $\alpha_{2}(x, y, \tau)$ be that of

$$
\alpha(x, y, \tau)=k(y,-x)+\int_{\bar{\Omega}_{\mathrm{r}}} k(y, z) \alpha(x, z, \tau) \mathrm{d} z .
$$

Since $K_{z}$ is a compact self adjoint operator on $L_{2}\left(\bar{\Omega}_{z}\right)$ it follows from the general theory of such operators that $K_{\tau}$ has a discrete set of real eigenvalues $\left\{\lambda_{i}(\tau)\right\}_{i=1}^{\infty}$ with the origin as the only possible limit point.

The main results we prove are Theorems 1.1 and 1.2 below:

Theorem 1.1. Suppose the domain satisfies conditions $\mathscr{C}_{1}-\mathscr{C}_{4}$ and the kernel, conditions $\mathscr{C}_{5}-\mathscr{C}_{7}$, and suppose that $\mathscr{C}_{8}$ holds. Then there exists a function $u(x, y, \tau)$ such that for each fixed $x \in \bar{\Omega}_{\tau}, u$ is continuous in the variable $y$ on $\bar{\Omega}_{\tau}$ and

$$
u(x, y, \tau)=\alpha_{1}(x, y, \tau)-k(y, x)
$$

in the $L_{2}$ sense as a function in the variable $y$ on $\bar{\Omega}_{\tau}$ and the corresponding Kac-Akhiezer formula is given by

$$
\exp \left\{-\int_{t=0}^{\tau} \mathrm{d} t \int_{\partial \bar{\Omega}_{1}} u(x, x, t) \mathrm{d} \sigma_{t}(x)\right\}=\prod_{i=1}^{\infty}\left[\exp \left(\lambda_{i}(\tau)\right)\right]\left[1-\lambda_{i}(\tau)\right]
$$

If $k$ satisfies the conditions $\mathscr{C}_{1}-\mathscr{C}_{7}$, we can apply the Theorem 1.1 to the kernel

$$
g_{\lambda}(x, y) \stackrel{\text { def }}{=} \lambda k(x, y) \quad(\lambda \in \mathbb{R})
$$

if $1 / \lambda$ is not in the spectrum of $K_{r}$. For the kernel $g_{\lambda}$ the eigenvalues will be $\left\{\lambda \lambda_{n}(\tau)\right\}_{n=1}^{\infty}$ and the corresponding Kac-Akhiezer formula is given by

$$
\begin{aligned}
\delta_{1}(\lambda, \tau) & \stackrel{\text { def }}{=} \exp \left\{-\int_{t=0}^{\tau} \mathrm{d} t \int_{\partial \bar{\Omega}_{\tau}} u(x, x, t ; \lambda) \mathrm{d} \sigma_{t}(x)\right\} \\
& =\prod_{i=1}^{\infty}\left[\exp \left(\lambda \lambda_{i}(\tau)\right)\right]\left[1-\lambda \lambda_{i}(\tau)\right],
\end{aligned}
$$

where $u(\cdot, ; t ; \lambda)$ is as in (1.4) with $k$ replaced by $g_{\lambda}$ and $\alpha_{1}$ is the solution of (1.2) with $k$ replaced by $g_{\lambda}$.

It is to be noted that $\delta_{1}(\lambda, \tau)$ has an analytic extension in the complex $\lambda$-plane. It follows from (1.7) that $\lambda_{0} \in \mathbb{R}^{1} \backslash\{0\}$ is an eigenvalue of $K_{\tau}$ if and only if $1 / \lambda_{0}$ is a zero of this extended function.

Apart from the conditions $\mathscr{C}_{1}-\mathscr{C}_{8}$ if the $\Omega_{\tau}$ 's are symmetric for $\tau>0$ and the kernel $k$ is real valued. we prove 
Theorem 1.2. Suppose as in Theorem 1.1, conditions $\mathscr{C}_{1}-\mathscr{C}_{8}$ hold and in addition the domains $\Omega_{\tau}($ for $\tau>0)$ are symmetric and $k$ is real valued. Then there exists a function $v(x, y, \tau)$ such that for each fixed $x \in \bar{\Omega}_{\tau}$, $v$ is continuous in the variable $y$ on $\bar{\Omega}_{\tau}$ and

$$
v(x, y, \tau)=\alpha_{2}(x, y, \tau)-k(y,-x)
$$

in the $L_{2}$ sense as a function in the variable $y$ on $\bar{\Omega}_{\tau}$ and the corresponding Kac-Akhiezer formula is given by

$$
\exp \left\{-\int_{t=0}^{\tau} \mathrm{d} t \int_{\partial \bar{\Omega}_{t}} v(x, x, t) \mathrm{d} \sigma_{t}(x)\right\}=\frac{\Pi_{i}^{+}\left[\exp \left(\lambda_{i}(\tau)\right)\right]\left[1-\lambda_{i}(\tau)\right]}{\Pi_{j}^{-}\left[\exp \left(\lambda_{j}(\tau)\right)\right]\left[1-\lambda_{j}(\tau)\right]},
$$

where $\Pi_{i}^{+}$and $\Pi_{j}^{-}$are products over all eigenvalues that have symmetric and antisymmetric eigenfunctions respectively.

Consider the kernel $k$ and the family $\left\{\bar{\Omega}_{\tau}\right\}_{\tau} \geqslant 0$ as in Theorem 1.2. We can apply Theorem 1.2 to $g_{\lambda}(x, y)$. For this kernel the eigenvalues will be $\left\{\lambda \lambda_{n}(\tau)\right\}_{n=1}^{\infty}$ and the corresponding Kac-Akhiezer formula is given by

$$
\begin{aligned}
\delta_{2}(\lambda, \tau) & \stackrel{\text { def }}{=} \exp \left\{-\int_{t=0}^{\tau} \mathrm{d} t \int_{\partial \bar{\Omega}_{t}} v(x, x, t ; \lambda) \mathrm{d} \sigma_{t}(x)\right\} \\
& =\frac{\Pi_{i}^{+}\left[\exp \left(\lambda \lambda_{i}(\tau)\right)\right]\left[1-\lambda \lambda_{i}(\tau)\right]}{\Pi_{j}^{-}\left[\exp \left(\lambda \lambda_{j}(\tau)\right)\right]\left[1-\lambda \lambda_{j}(\tau)\right]},
\end{aligned}
$$

where $v(\cdot, ; t ; \lambda)$ is as in (1.8) with $k$ replaced by $g_{\lambda}$ and $\alpha_{2}$ is the solution of (1.3) with $k$ replaced by $g_{\lambda}$.

It is to be noted that $\delta_{2}(\lambda, \tau)$ has a meromorphic extension in the complex $\lambda$-plane. It follows from (1.10) that $\lambda_{0}(\neq 0) \in \mathbb{R}^{1}$ is an eigenvalue of $K_{\tau}$ if and only if $1 / \lambda_{0}$ is a zero or a pole of this function.

We observe that Theorem 1.1 is the generalization of the classical Kac-Akhiezer formula (1.1) as extended by Vittal Rao [8] for kernels continuous near the origin and Theorem 1.2 is the generalization of the meromorphic function extension obtained by Vittal Rao [8].

\section{Some properties of the eigenvalues}

We now obtain some properties of the eigenvalues and eigenfunctions similar to those obtained by Vittal Rao [7]. The ideas behind the proofs are similar to those in [7].

Let $\lambda_{i}^{+}(\tau)$ and $\lambda_{i}^{-}(\tau)$ be respectively the $i$ th positive and negative eigenvalues of $K_{\tau}$. By the minimax characterization [6], [10] of the eigenvalues we have

Lemma 2.1. For each $i \in \mathbb{N}, \lambda_{i}^{+}(\tau)$ and $\lambda_{i}^{-}(\tau)$ are respectively nondecreasing and nonincreasing functions for $\tau \geqslant 0$ if the $\Omega_{\tau}$ 's satisfy the conditions $\mathscr{C}_{1}-\mathscr{C}_{2}$ and the kernel $k$ satisfies the conditions $\mathscr{C}_{5}-\mathscr{C}_{6}$.

Lemma 2.2. $\lambda_{i}(\tau)$ is an absolutely continuous function in the variable $\tau$ in every bounded 
interval of $[0, \infty)$ for each $i \in \mathbb{N}$, if $k$ is a locally integrable symmetric kernel on $\mathbb{R}^{n} \times \mathbb{R}^{n}$ and the $\Omega_{\tau}$ 's satisfy the conditions $\mathscr{C}_{1}-\mathscr{C}_{3}$.

Proof. For $\tau \geqslant 0$ we define

$$
F(\tau)=\int_{\bar{\Omega}_{\tau}} \int_{\bar{\Omega}_{\tau}}|k(x, y)|^{2} \mathrm{~d} x \mathrm{~d} y .
$$

For a $\tau_{0} \in(0, \infty)$ and a finite sequence of disjoint open intervals $\left\{\left(\tau_{j}, \eta_{j}\right)\right\}_{j=1}^{m}$ in $\left[0, \tau_{0}\right]$, we have

and

$$
\begin{aligned}
& \sum_{j=1}^{m} F\left(\eta_{j}\right)-F\left(\tau_{j}\right) \\
& =\sum_{j=1}^{m}\left\{\int_{\bar{\Omega}_{n,}} \mathrm{~d} x \int_{\bar{\Omega}_{n,}, \bar{\Omega}_{, j}}|k(x, y)|^{2} \mathrm{~d} y+\int_{\bar{\Omega}_{n,}, \bar{\Omega}_{,}} \mathrm{d} x \int_{\bar{\Omega}_{\mathrm{r}}}|k(x, y)|^{2} \mathrm{~d} y\right\} \\
& \leqslant \sum_{j=1}^{m}\left\{\int_{\bar{\Omega}_{\mathrm{ro}}} \mathrm{d} x \int_{\bar{\Omega}_{\eta_{j}} \backslash \bar{\Omega}_{\mathrm{r}}}|k(x, y)|^{2} \mathrm{~d} y+\int_{\bar{\Omega}_{\eta,}, \bar{\Omega}_{z_{s}}} \mathrm{~d} x \int_{\bar{\Omega}_{\mathrm{z}_{0}}}|k(x, y)|^{2} \mathrm{~d} y\right\} \\
& =2 \int_{\bar{\Omega}_{r o}} \int_{\left.\cup_{j=1}^{m}\left(\bar{\Omega}_{n j}\right\rangle \bar{\Omega}_{v y}\right)}|k(x, y)|^{2} \mathrm{~d} x \mathrm{~d} y
\end{aligned}
$$

$$
(\mu \times \mu)\left[\bar{\Omega}_{\tau_{0}} \times \cup_{j=1}^{m}\left(\bar{\Omega}_{n_{j}} \backslash \bar{\Omega}_{\tau_{j}}\right)\right]=\mu\left(\bar{\Omega}_{\tau_{0}}\right) \sum_{j=1}^{m}\left[\mu\left(\bar{\Omega}_{\eta_{j}}\right)-\mu\left(\bar{\Omega}_{\tau_{j}}\right)\right]
$$

Let $v$ be the measure defined on the elementary sets as

$$
v(E \times F)=\iint_{E \times F}|k(x, y)|^{2} \mathrm{~d} x \mathrm{~d} y .
$$

Since the map $\tau \mapsto \mapsto \mu\left(\bar{\Omega}_{\tau_{0}}\right) \mu\left(\vec{\Omega}_{\tau}\right)$ is absolutely continuous in $\left[0, \tau_{0}\right]$ and $\nu \ll \mu \times \mu$, using (2.2) and (2.3) one has $F(\tau)$ is absolutely continuous in the interval $\left[0, \tau_{0}\right]$. From $(2.1)$ and the classical theory of H-S operators [6], we have

$$
F(\tau)=\sum_{i=1}^{\infty} \lambda_{i}^{2}(\tau)
$$

hence by Lemma 2.1 , we have for $i \in \mathbb{N}$, and $j=1,2,3, \ldots, m$,

$$
F\left(\eta_{j}\right)-F\left(\tau_{j}\right) \geqslant \lambda_{i}^{2}\left(\eta_{j}\right)-\lambda_{i}^{2}\left(\tau_{j}\right) \geqslant 0,
$$

from which it follows that $\lambda_{i}^{2}(\tau)$ is an absolutely continuous function in the variable $\tau$ in the interval $\left[0, \tau_{0}\right]$. Hence by Lemma 2.1 we have $\lambda_{i}^{+}(\tau)$ is absolutely continuous in the interval $\left[0, \tau_{0}\right]$. By considering $-K_{\tau}$ instead of $K_{\tau}$ one can conclude the same for the negative eigenvalues. This completes the proof of the lemma.

Lemma 2.1 implies that for each $i \in \mathbb{N}, \lambda_{i}(\tau)$ is differentiable almost everywhere. Let us denote an eigenfunction corresponding to an eigenvalue $\lambda_{i}(\tau)$ of $K_{\tau}$ by $\phi_{i}(\cdot, \tau)$. 
Lemma 2.3 Let $i \in \mathbb{N}$ be fixed and $\lambda_{i}(\tau)$ be of multiplicity $m_{i}(\tau)$. Then there exist $m_{i}(\tau)$ orthonormal eigenfunctions $\left\{\phi_{i j}(\cdot, \tau)\right\}_{j=1}^{m_{1}(\tau)}$ such that

$$
\frac{\mathrm{d} \lambda_{i}(\tau)}{\mathrm{d} \tau}=\lambda_{i}(\tau) \int_{\partial \bar{\Omega}_{\tau}}\left|\phi_{i j}(x, \tau)\right|^{2} \mathrm{~d} \sigma_{\tau}(x) ; j=1,2, \ldots, m_{i}(\tau),
$$

for almost every $\tau$, if the $\Omega_{\tau}$ and $k$ satisfy the conditions $\mathscr{C}_{1}-\mathscr{C}_{7}$.

Proof. It is enough if we prove the result for the positive eigenvalues of $K_{\tau}$. Let $\tilde{\tau} \geqslant 0$ be such that $\lambda_{i}^{+}(\tau)=0$ for all $\tau \leqslant \tilde{\tau}$ and $\lambda_{i}^{+}(\tau)>0$ for $\tau>\tilde{\tau}$. Hence (2.4) is true for almost every $\tau \leqslant \tilde{\tau}$ and for any choice of eigenfunctions. Now $\lambda_{i}^{+}(\tau)$ and $\mu\left(\bar{\Omega}_{\mathfrak{r}}\right)$ are differentiable almost everywhere by Lemma 2.1 and assumption $\mathscr{C}_{3}$ respectively. Let $\tau_{0}>\tilde{\tau}$ be such that both $\mathrm{d} \lambda_{i}^{+}(\tau) / \mathrm{d} \tau$ and $\mathrm{d} \mu\left(\bar{\Omega}_{\tau}\right) / \mathrm{d} \tau$ exist at $\tau=\tau_{0}$. Let $\left\{\tau_{p}\right\}_{p=1}^{\infty}$ be an increasing sequence in the interval $\left(\tilde{\tau}, \tau_{0}\right)$ such that

$$
\tau_{p} \rightarrow \tau_{0} \text { as } p \rightarrow \infty
$$

First let us consider the case when the multiplicity of $\lambda_{i}^{+}\left(\tau_{0}\right)$ namely $m_{i}\left(\tau_{0}\right)$ is one. Consider the sequence of eigenvalues $\left\{\lambda_{i}\left(\tau_{p}\right)\right\}_{p=1}^{\infty}$ and a corresponding sequence $\left\{\phi_{i}\left(\cdot, \tau_{p}\right)\right\}_{p=1}^{\infty}$ of normalized eigenfunctions of $K_{\tau_{p}}$.

We have

and

$$
\lambda_{i}^{+}\left(\tau_{p}\right) \phi_{i}\left(x, \tau_{p}\right)=\int_{\bar{\Omega}_{\mathrm{s}_{p}}} k(x, y) \phi_{i}\left(y, \tau_{p}\right) \mathrm{d} y
$$

$$
\int_{\bar{\Omega}_{i p}}\left|\phi_{i}\left(x, \tau_{p}\right)\right|^{2} \mathrm{~d} x=1
$$

Though initially the eigenfunctions $\phi_{i}\left(x, \tau_{p}\right)$ are defined only in $\bar{\Omega}_{\tau_{p}}$, the right hand side of (2.5) allows us to extend $\phi_{i}\left(x, \tau_{p}\right)$ for all $x \in \mathbb{R}^{n}$. Hence without loss of generality we can take each one of the $\phi_{i}\left(\cdot, \tau_{p}\right)$ to be defined on $\bar{\Omega}_{\tau_{0}}$.

For each $x \in \bar{\Omega}_{\tau}$, we define a linear functional $F_{x, \tau}$ on $L_{2}\left(\bar{\Omega}_{\tau}\right)$ by

$$
F_{x, \mathrm{r}}(f)=\int_{\bar{\Omega}_{\mathrm{r}}} k(x, y) f(y) \mathrm{d} y .
$$

Condition $\mathscr{C}_{7}$ implies that the map $x \mapsto k_{x}$ from $\bar{\Omega}_{\tau}$ to $L_{2}\left(\bar{\Omega}_{\tau}\right)$ is weakly continuous; and hence $\left\{k_{x}\right\}_{x \in \bar{\Omega}_{\tau}}$ is a weakly compact subset of $L_{2}\left(\bar{\Omega}_{\tau}\right)$. Hence for $f \in L_{2}\left(\bar{\Omega}_{\tau}\right)$, there exists a constant $c_{f, \tau} \in(0, \infty)$ such that

$$
\sup _{x \in \bar{\Omega}_{\tau}}\left|\int_{\bar{\Omega}_{\tau}} k_{x}(y) f(y) \mathrm{d} y\right| \leqslant c_{f, \tau},
$$

which implies that

$$
\sup _{x \in \bar{\Omega}}\left|F_{x, \tau}(f)\right| \leqslant c_{f, \tau}
$$

Hence by uniform boundedness principle one can choose a constant $M_{\tau} \in(0, \infty)$ such that

$$
\left\|F_{x, \tau}\right\| \leqslant M_{\tau}, \quad \forall x \in \bar{\Omega}_{\tau}
$$


Since

$$
\left\|F_{x, \tau}\right\|^{2}=\int_{\bar{\Omega}_{\tau}}|k(x, y)|^{2} \mathrm{~d} y,
$$

it now follows from (2.7) that,

$$
\begin{aligned}
& \forall \tau>0, \text { there exists a constant } M_{\tau} \in(0, \infty) \text { such that } \\
& \int_{\bar{\Omega}_{\tau}}|k(x, y)|^{2} \mathrm{~d} y \leqslant M_{\tau}^{2}, \quad \forall x \in \bar{\Omega}_{\tau} .
\end{aligned}
$$

We now prove the following properties of the eigenvalue sequence $\left\{\phi_{i}\left(\cdot, \tau_{p}\right)\right\}_{p=1}^{\infty}$ :

(a) $\phi_{i}\left(\cdot, \tau_{p}\right) \in C^{0}\left(\bar{\Omega}_{\tau_{0}}\right), \forall p$.

(b) There exists a $J_{i} \in[0, \infty)$ such that $\left|\phi_{i}\left(x, \tau_{p}\right)\right| \leqslant J_{i}, \forall x \in \bar{\Omega}_{\tau_{0}}$ and $\forall p$.

(c) There exists a subsequence $\left\{\phi_{i}\left(\cdot, \tau_{q}\right)\right\}_{q=1}^{\infty}$ which converges uniformly on $\bar{\Omega}_{\text {ro }}$.

Proof of $(a)$ : For each $p$, by $\mathscr{C}_{7}$, the map

$$
x \mapsto \int_{\bar{\Omega}_{\mathrm{t}}} k(x, y) \phi_{i}\left(y, \tau_{p}\right) \mathrm{d} y,
$$

from the compact set $\bar{\Omega}_{\tau_{0}}$ into $\mathbb{C}$ is continuous; hence by (2.5) the map

$$
x \mapsto \lambda_{i}^{+}\left(\tau_{p}\right) \phi_{i}\left(x, \tau_{p}\right)
$$

from $\bar{\Omega}_{\tau_{0}}$ into $\mathbb{C}$ is continuous. Now (a) follows as $\lambda_{i}^{+}\left(\tau_{p}\right)>0$.

Proof of $(b)$ : Applying Schwartz inequality to the right hand side of (2.5) and using (2.6) we get

$$
\left|\lambda_{i}^{+}\left(\tau_{p}\right) \phi_{i}\left(x, \tau_{p}\right)\right|^{2} \leqslant \int_{\bar{\Omega}_{\tau_{p}}}|k(x, y)|^{2} \mathrm{~d} y \quad \forall x \in \bar{\Omega}_{\tau_{0}} \text { and } \forall p
$$

Now using $\mathscr{C}_{2}$ and (2.12) we get

$$
\left|\lambda_{i}^{+}\left(\tau_{p}\right) \phi_{i}\left(x, \tau_{p}\right)\right| \leqslant M_{\tau_{0}}, \quad \forall x \in \bar{\Omega}_{\tau_{0}} \text { and } \forall p
$$

where $M_{t_{0}}$ is as given in (2.8). Since $\lambda_{i}^{+}\left(\tau_{1}\right)>0$, (b) follows by Lemma 2.1 .

Proof of $(c)$ : We shall do this in two steps.

Step 1. By (b) and Banach-Alaoglu theorem one can find a sub sequence $\left\{\phi_{i}\left(\cdot, \tau_{q}\right)\right\}_{q=1}^{\infty}$ of $\left\{\phi_{i}\left(\cdot, \tau_{p}\right)\right\}_{p=1}^{\infty}$ and a $\psi_{i} \in L_{2}\left(\bar{\Omega}_{z_{0}}\right)$ such that $\phi_{i}\left(\cdot, \tau_{q}\right) \rightarrow \psi_{i}$ weakly in $L_{2}\left(\bar{\Omega}_{0}\right)$ as $q \rightarrow \infty$. Since the operator $K_{t_{0}}: L_{2}\left(\bar{\Omega}_{\tau_{0}}\right) \rightarrow L_{2}\left(\bar{\Omega}_{\tau_{0}}\right)$ is compact, it now follows that

$$
\left\|K_{\tau_{0}} \phi_{i}\left(\cdot, \tau_{q}\right)-K_{\tau_{0}} \psi_{i}\right\|_{L_{2}\left(\bar{\Omega}_{z_{0}}\right)} \rightarrow 0 \text { as } q \rightarrow \infty \text {. }
$$


We see that

$$
\begin{aligned}
& \left\|\int_{\bar{\Omega}_{\tau_{q}}} k(\cdot, y) \phi_{i}\left(y, \tau_{q}\right) \mathrm{d} y-\int_{\bar{\Omega}_{\tau_{0}}} k(\cdot, y) \psi_{i}(y) \mathrm{d} y\right\|_{L_{2}\left(\bar{\Omega}_{\tau_{0}}\right)} \\
& \quad \leqslant\left\|\int_{\bar{\Omega}_{\tau_{0}} \backslash \bar{\Omega}_{\tau_{q}}} k(\cdot, y) \phi_{i}\left(y, \tau_{q}\right) \mathrm{d} y\right\|_{L_{2}\left(\bar{\Omega}_{\mathrm{r}}\right)}+\left\|\int_{\bar{\Omega}_{\tau_{0}}} k(\cdot, y)\left[\phi_{i}\left(y, \tau_{q}\right)-\psi_{i}(y)\right] \mathrm{d} y\right\|_{L_{2}\left(\bar{\Omega}_{\tau_{0}}\right)} \\
& \quad \leqslant\left(\int_{\bar{\Omega}_{\tau_{0}}} \int_{\bar{\Omega}_{\mathrm{r}_{0}} \bar{\Omega}_{\tau_{q}}}|k(x, y)|^{2} \mathrm{~d} x \mathrm{~d} y\right)^{1 / 2}\left[\mu\left(\bar{\Omega}_{\tau_{0}} \bar{\Omega}_{\tau_{q}}\right)\right]^{1 / 2} J_{i}+\left\|K_{\tau_{0}} \phi_{i}\left(\cdot, \tau_{q}\right)-K_{\tau_{0}} \psi_{i}\right\|_{L_{2}\left(\bar{\Omega}_{\mathrm{ro}}\right)},
\end{aligned}
$$

where $J_{i}$ is as given in (2.10). Hence we have

$$
\left\|\frac{1}{\lambda_{i}^{+}\left(\tau_{q}\right)} \int_{\bar{\Omega}_{\tau_{0}}} k(\cdot, y) \phi_{i}\left(y, \tau_{q}\right) d y-\frac{1}{\lambda_{i}^{+}\left(\tau_{0}\right)} \int_{\bar{\Omega}_{\tau_{0}}} k(\cdot, y) \psi_{i}(y) d y\right\|_{L_{2}\left(\bar{\Omega}_{\mathrm{r}_{0}}\right)} \rightarrow 0 \text { as } q \rightarrow \infty ;
$$

from which it follows that

and

$$
\psi_{i}=\frac{1}{\lambda_{i}^{+}\left(\tau_{0}\right)} K_{\tau_{0}} \psi_{i}
$$

$$
\left\|\phi_{i}\left(\cdot, \tau_{q}\right)-\psi_{i}\right\|_{L_{2}\left(\bar{\Omega}_{\mathrm{ro}_{0}}\right)} \rightarrow 0 \text { as } q \rightarrow \infty .
$$

From (2.6) it follows that

As

$$
1=\int_{\bar{\Omega}_{\tau 0}}\left|\phi_{i}\left(x, \tau_{q}\right)\right|^{2} \mathrm{~d} x+\int_{\bar{\Omega}_{\tau_{0}} \backslash \bar{\Omega}_{\mathrm{rq}}} \mid \phi_{i}\left(x, \tau_{q}\right)^{2} \mathrm{~d} x \quad \forall q .
$$

$$
\mu\left(\overline{\boldsymbol{\Omega}}_{\tau_{0}} \backslash \overline{\boldsymbol{\Omega}}_{\tau_{q}}\right) \rightarrow 0 \text { when } q \rightarrow \infty,
$$

using (2.10) in (2.15) we get

$$
\lim _{q \rightarrow \infty} \int_{\bar{\Omega}_{\tau 0}}\left|\phi_{i}\left(x, \tau_{q}\right)\right|^{2} \mathrm{~d} x=1
$$

Since the map $f \mapsto\|f\|$ from $L_{2}\left(\bar{\Omega}_{\tau_{0}}\right)$ to $\mathbb{R}^{1}$ is continuous, from (2.14) and (2.17) it follows that

$$
\int_{\bar{\Omega}_{\tau_{0}}}\left|\psi_{i}(x)\right|^{2} \mathrm{~d} x=1
$$

From (2.13) and (2.18) we have $\psi_{i}$ is a normalized eigenfunction of $K_{t_{0}}$ corresponding to the eigenvalue $\lambda_{i}^{+}\left(\tau_{q}\right)$.

Step 2. Let us denote the $\psi_{i}$ by $\phi_{i}\left(\cdot, \tau_{0}\right)$. Then $(2.13)$ can be written as

$$
\lambda_{i}^{+}\left(\tau_{0}\right) \phi_{i}\left(x, \tau_{0}\right)=\int_{\bar{\Omega}_{\mathrm{r}}} k(x, y) \phi_{i}\left(y, \tau_{0}\right) \mathrm{d} y .
$$


From (2.5) and (2.19) it follows that for any $x \in \overline{\mathbf{\Omega}}_{r_{0}}$,

$$
\begin{aligned}
& \left|\lambda_{i}^{+}\left(\tau_{q}\right) \phi_{i}\left(x, \tau_{q}\right)-\lambda_{i}^{+}\left(\tau_{0}\right) \phi_{i}\left(x, \tau_{0}\right)\right| \\
& \leqslant\left(\int_{\bar{\Omega}_{\mathrm{i} \mid} \mid \bar{\Omega}_{\mathrm{r}}}|k(x, y)|^{2} \mathrm{~d} y\right)^{1 / 2}\left(\int_{\bar{\Omega}_{\mathrm{i}} \mid \bar{\Omega}_{\mathrm{i}}}\left|\phi_{i}\left(y, \tau_{0}\right)\right|^{2} \mathrm{~d} y\right)^{1 / 2} \\
& +\left(\int_{\bar{\Omega}_{\mathrm{q}}}|k(x, y)|^{2} \mathrm{~d} y\right)^{1 / 2}\left(\int_{\bar{\Omega}_{\mathrm{q}}}\left|\phi_{i}\left(y, \tau_{q}\right)-\phi_{i}\left(y, \tau_{0}\right)\right|^{2} \mathrm{~d} y\right)^{1 / 2} \\
& \leqslant M_{\tau_{0}} J_{i}\left[\mu\left(\bar{\Omega}_{\tau_{0}} \backslash \bar{\Omega}_{\tau_{q}}\right)\right]^{1 / 2}+M_{\tau_{0}}^{1 / 2}\left\|\phi_{i}\left(\cdot, \tau_{q}\right)-\phi_{i}\left(\cdot, \tau_{0}\right)\right\|_{L_{2}\left(\bar{\Omega}_{\tau_{0}}\right)} \cdot
\end{aligned}
$$

Now (2.14) and (2.16) imply that

$$
\phi_{i}\left(x, \tau_{q}\right) \rightarrow \phi_{i}\left(x, \tau_{0}\right) \text { uniformly on } \bar{\Omega}_{\tau_{0}} \text { as } q \rightarrow \infty .
$$

This completes the proof of (c).

From $(2.5)$ and $\left(\mathscr{C}_{4}\right.$ ii) it can be easily seen that

$$
\begin{aligned}
& \frac{\lambda_{i}^{+}\left(\tau_{0}\right)-\lambda_{i}^{+}\left(\tau_{q}\right)}{\tau_{0}-\tau_{q}} \int_{\bar{\Omega}_{\tau_{0}}} \phi_{i}\left(x, \tau_{q}\right) \overline{\phi_{i}\left(x, \tau_{0}\right)} \mathrm{d} x \\
& =\frac{\lambda_{i}^{+}\left(\tau_{0}\right)}{\tau_{0}-\tau_{q}} \int_{\bar{\Omega}_{\mathrm{t}}\left(\overline{\Omega_{i}}\right.}\left\{\phi_{i}\left(y, \tau_{q}\right)-\phi_{i}\left(x, \tau_{0}\right)\right\} \overline{\phi_{i}\left(y, \tau_{0}\right)} \mathrm{d} y \\
& \quad+\frac{\lambda_{i}^{+}\left(\tau_{0}\right)}{\tau_{0}-\tau_{q}} \int_{\bar{\Omega}_{\tau_{0}} \backslash \bar{\Omega}_{\mathrm{sq}}}\left|\phi_{i}\left(y, \tau_{0}\right)\right|^{2} \mathrm{~d} y \\
& =\frac{\lambda_{i}^{+}\left(\tau_{0}\right)}{\tau_{0}-\tau_{q}}\left[\int_{t=\tau_{q}}^{\tau_{0}} \int_{\partial \bar{\Omega}_{i}}\left\{\phi_{i}\left(y, \tau_{q}\right)-\phi_{i}\left(y, \tau_{0}\right)\right\} \overline{\phi_{i}\left(y, \tau_{0}\right)} \mathrm{d} \sigma_{t}(y)\right. \\
& \left.\quad+\int_{\bar{\Omega}_{\tau_{0}} \backslash \bar{\Omega}_{i}}\left|\phi_{i}\left(y, \tau_{0}\right)\right|^{2} \mathrm{~d} y\right] .
\end{aligned}
$$

Letting $q \rightarrow \infty$ and using (c) and $\left(\mathscr{C}_{4} \mathrm{i}\right)$, we get (2.4). This proof can be easily modified for the case $m_{i}\left(\tau_{0}\right)>1$ along similar lines as in [7].

\section{Proofs of the main results}

Proof of Theorem 1.1: (2.8) implies that the series $\Sigma_{i=1}^{\infty} \lambda_{i}(\tau)\left\langle\alpha_{1}, \phi_{i}\right\rangle \phi_{i}(y, \tau)$ converges uniformly and absolutely $[6]$ to $\int_{\bar{\Omega}_{\mathrm{s}}} k(y, z) \alpha_{1}(x, z, \tau) \mathrm{d} z$ where

$$
\left\langle\alpha_{1}, \phi_{i}\right\rangle=\int_{\bar{\Omega}_{\tau}} \alpha_{1}(x, z, \tau) \overline{\phi_{i}(z, \tau)} \mathrm{d} z .
$$

Now using (1.2) we get

$$
\alpha_{1}(x, y, \tau)-k(y, x)=\sum_{i=1}^{\infty} \lambda_{i}(\tau)\left\langle\alpha_{1}, \phi_{i}\right\rangle \phi_{i}(y, \tau) .
$$


From the above equation one easily gets

$$
\left\langle\alpha_{1}, \phi_{i}\right\rangle=\frac{\lambda_{i}(\tau)}{1-\lambda_{i}(\tau)} \overline{\phi_{i}(x, \tau)}
$$

Let $u(x, y, \tau)=\alpha_{1}(x, y, \tau)-k(y, x)$. We have from (3.1) and (3.2)

$$
u(x, y, \tau)=\sum_{i=1}^{\infty} \frac{\left(\lambda_{i}(\tau)\right)^{2}}{1-\lambda_{i}(\tau)} \phi_{i}(y, \tau) \overline{\phi_{i}(x, \tau)}
$$

For each fixed $x \in \bar{\Omega}_{\tau}$, the series in the right hand side of (3.3) converges uniformly and ablolutely in the variable $y$ and hence the sum is a continuous function in $y$. It therefore follows that $u(x, y, \tau)$ has a continuous representation. Letting $y \rightarrow x$ in (3.3) and using monotone convergence theorem and Lemma 2.3 we get

$$
\int_{\partial \bar{\Omega}_{i}} u(x, x, \tau) \mathrm{d} \sigma_{\tau}(x)=\sum_{i=1}^{\infty} \frac{\lambda_{i}(\tau)}{1-\lambda_{i}(\tau)} \frac{\mathrm{d} \lambda_{i}(\tau)}{\mathrm{d} \tau} .
$$

By Lemma 2.2 as in [8] we get

$$
-\int_{t=0}^{\tau} \mathrm{d} t \int_{\partial \bar{\Omega}_{i}} u(x, x, t) \mathrm{d} \sigma_{t}(x)=\sum_{i=1}^{\infty}\left\{\lambda_{i}(\tau)+\log \left[1-\lambda_{i}(\tau)\right]\right\} .
$$

Now (1.5) is easily obtained by exponentiating both sides.

Proof of Theorem 1.2: Since the kernel is real valued, one can choose $\phi_{i}$ 's such that they are real and

$$
\phi_{i}(-x, \tau)= \pm \phi_{i}(x, \tau), \quad \forall x \in \bar{\Omega}_{\tau}
$$

where \pm correspond respectively to those eigenvalues having symmetric and skew symmetric eigenfunction. By using arguments as in the proof of Theorem 1.1, one can prove that

$$
v(x, y, \tau)=\sum_{i=1}^{\infty} \frac{\left(\lambda_{i}(\tau)\right)^{2}}{1-\lambda_{i}(\tau)} \phi_{i}(y, \tau) \overline{\phi_{i}(-x, \tau)}
$$

where $v(x, y, \tau)$ is a continuous function on $\bar{\Omega}_{\tau}$ in the variable $y$ for each fixed $x \in \overline{\mathbf{\Omega}}_{\tau}$. Letting $y \rightarrow x$ in (3.5) and using (3.4) we get

$$
v(x, x, \tau)= \pm \sum_{i=1}^{\infty} \frac{\left(\lambda_{i}(\tau)\right)^{2}}{1-\lambda_{i}(\tau)}\left|\phi_{i}(x, \tau)\right|^{2}
$$

Now by arguments as in the proof of Theorem 1.1 one can arrive at (1.9).

\section{Some remarks}

Remark 1. (Non symmetric case): In all the above lemmas if the kernel is not symmetric then the analogous results hold for the singular numbers, $s_{i}(\tau)$. That is, we get for 
each $i=1,2,3, \ldots$,

(1). $s_{i}(\tau)$ is a nondecreasing function in $\tau$,

(2). $s_{i}(\tau)$ is absolutely continuous in each bounded interval of $[0, \infty)$,

(3). There exist $m_{i}(\tau)$ orthonormal eigenfunctions $\phi_{i j}(\cdot, \tau) \in L_{2}\left(\Omega_{\varepsilon}\right)$ corresponding to the eigenvalue $s_{i}^{2}(\tau)$ of $K_{\tau}^{*} K_{\tau}$ such that

$$
\frac{\mathrm{d} s_{i}(\tau)}{\mathrm{d} \tau}=\frac{1}{2} s_{i}(\tau) \int_{\partial \bar{\Omega}_{\mathrm{r}}}\left|\phi_{i j}(x, \tau)\right|^{2} \mathrm{~d} \sigma_{\tau}(x) \quad \text { for } j=1,2,3, \ldots m_{i}(\tau),
$$

and for almost every $\tau \geqslant 0$; where $m_{i}(\tau)$ is the multiplicity of the eigenvalue $s_{i}^{2}(\tau)$.

Remark 2. Lemma 2.3, Theorems 1.1 and 1.2 as well as their proofs remain valid even if we replace the condition $\mathscr{C}_{7}$ by a weaker one given below:

Given two compact subsets $E$ and $F$ of $\mathbb{R}^{n}$ the map $x \mapsto k_{x}$ from $E$ to $L_{2}(F)$ is weakly continuous, meaning that,

"for each $f \in L_{2}(F)$, the map $x \mapsto \int_{F} k_{x}(y) \overline{f(y)} \mathrm{d} y$ from $E$ to $\mathbb{C}$ is continuous."

\section{Acknowledgements}

The authors are thankful to Dr S Ramaswamy, TIFR Centre, Bangalore, several discussions with whom have helped to sharpen some of the results. The authors thank Prof. M S Ramanujan, Department of Mathematics, University of Michigan, Ann Arbor for his useful comments.

\section{References}

[1] Akhiezer N I, A continual analogue of some theorems on Toeplitz matrices, AMS Transl., 50 (1966) 295-316

[2] Dunford N and Schwartz J T, Linear operators, Vol II, (Interscience) 1963

[3] Kac M, Toeplitz-matrices, translation kernels and a related problem in probability theory, Duke Math. J., 21 (1954) 501-509

[4] Kac M, Theory and application of Toeplitz forms, in Summer institute on spectral theory and statistical mechanics, (Brookhaven National Laboratory), (1965) pp. 1-56

[5] Mullikin T W and Vittal Rao R, Extended Kac-Akhiezer formula for the Fredholm determinant of integral operators, J. Math. Anal. Appl., 61 (1977) 409-415

[6] Riesz F and Nagy Sz, Functional analysis, (Unger, New York) (1955)

[7] Vittal Rao R, On the eigenvalues of the integral operators with difference kernels, J. Math. Anal. Appl. 53 (1976) 554-566

[8] Vittal Rao R, Extended Akhiezer formula for the Fredholm determinant of difference kernels, $J$. Math. Anal. Appl. 54 (1976) 79-88

[9] Vittal Rao R and Sukavanam N, Kac-Akhiezer formula for normal integral operators J. Math. Anal. Appl., 114 (1986) 458-467

[10] Zabraiko P P et al, Integral equations - a reference text. (Noordhoff international publishing, Leyden) 1975 